\title{
Evolutionary Origins of the Endowment Effect: Evidence from Hunter-Gatherers
}

\section{Citation}

Apicella, Coren L., Eduardo M. Azevedo, Nicholas A. Christakis, and James H. Fowler. 2014. "Evolutionary Origins of the Endowment Effect: Evidence from Hunter-Gatherers." American Economic Review 104 (6) (June): 1793-1805. doi:10.1257/aer.104.6.1793.

\section{Published Version}

doi:10.1257/aer.104.6.1793

\section{Permanent link}

http://nrs.harvard.edu/urn-3:HUL.InstRepos:33839947

\section{Terms of Use}

This article was downloaded from Harvard University's DASH repository, and is made available under the terms and conditions applicable to Other Posted Material, as set forth at http:// nrs.harvard.edu/urn-3:HUL.InstRepos:dash.current.terms-of-use\#LAA

\section{Share Your Story}

The Harvard community has made this article openly available.

Please share how this access benefits you. Submit a story.

\section{Accessibility}




\title{
Evolutionary Origins of the Endowment Effect: Evidence from Hunter-Gatherers ${ }^{\dagger}$
}

\author{
By Coren L. Apicella, Eduardo M. Azevedo, \\ Nicholas A. Christakis, and James H. Fowler**
}

\begin{abstract}
The endowment effect, the tendency to value possessions more than non-possessions, is a well-known departure from rational choice and has been replicated in numerous settings. We investigate the universality of the endowment effect, its evolutionary significance, and its dependence on environmental factors. We experimentally test for the endowment effect in an isolated and evolutionarily relevant population of hunter-gatherers, the Hadza Bushmen of Northern Tanzania. We find that Hadza living in isolated regions do not display the endowment effect, while Hadza living in a geographic region with increased exposure to modern society and markets do display the endowment effect. (JEL C93, D12, O15)
\end{abstract}

This paper uses a natural experiment in a hunter-gatherer population to provide evidence on three questions: the universality of a behavioral bias (the endowment effect), its dependence on cultural factors, and its evolutionary significance. Systematic deviations from rational behavior have been extensively documented, along with their importance for market outcomes. Yet, evidence on the three questions above is sparse, especially for biases in economic decisions. First, most experiments are conducted in industrialized societies, and in particular on college campuses, so biases could be the result of shared experiences as opposed to innate features of human behavior. Second, cross-cultural experiments typically compare very different groups, making it hard to attribute differences in behavior to a specific cultural factor due to omitted variable bias (Roth et al. 1991; Henrich et al. 2001, 2006). Third, while many contributions in economics have proposed evolutionary

\footnotetext{
* Apicella: Department of Psychology, University of Pennsylvania, 3720 Walnut Street, Philadelphia, PA 191046241 (e-mail: capicella@psych.upenn.edu); Azevedo: The Wharton School of the University of Pennsylvania, 3620 Locust Walk, SHDH 1455, Philadelphia, PA 19104 (e-mail: eazevedo@wharton.upenn.edu); Christakis: Departments of Sociology, Ecology and Evolutionary Biology, and Medicine, Yale University, Yale Institute for Network Science, PO Box 208263, New Haven, CT 06520-8263 (e-mail: nicholas.christakis@yale.edu); Fowler: Political Science Department and Medical Genetics Department, University of California, San Diego, Social Science Building 392, 9500 Gilman Drive \#0521, La Jolla, CA 92093-0521 (e-mail: fowler@ucsd.edu). We thank Andrei Shleifer, Jonathan Beauchamp, Andreas Fuster, Alvin Roth, and seminar participants at Harvard and Yale for helpful comments and suggestions. Special thanks goes to Audax Mabulla and Chris and Nani Schmeling for their assistance in Tanzania. Finally, we thank our research assistants Mika Peterson and Ibrahim Mabulla for their hard work. Research for this publication was supported by funding from the Science of Generosity Initiative of the University of Notre Dame and the John Templeton Foundation and by award P01-AG031093 from the National Institute on Aging. The authors declare that they have no relevant or material financial interests that relate to the research described in this paper.

${ }^{\dagger}$ Go to http://dx.doi.org/10.1257/aer.104.6.1793 to visit the article page for additional materials and author disclosure statement(s).
} 
explanations for economic behavior and biases (Rayo and Becker 2007; Robson and Samuelson 2009), these theories are seldom faced with empirical tests (Chen, Lakshminarayanan, and Santos 2006; Harbaugh, Krause, and Vesterlund 2001).

Our empirical setting is particularly well suited to address these three questions. We performed experiments testing for the endowment effect with one of the last hunter-gatherer populations on the planet, the Hadza Bushmen. The Hadza present a unique opportunity to test for the universality of the endowment effect, as they have a high degree of isolation from modern culture, residing in a remote region of Northern Tanzania. They are also an ideal population for investigating the evolutionary origins of human preferences because they live in a physical and social environment that best approximates the environment where humans spent the majority of their evolution, before the advent of agriculture (Marlowe 2010).

Crucially, although the Hadza remain relatively isolated, a subset of this population has had increased contact with modern society and markets. In the last few years there has been an increase in ethno-tourism with safari companies visiting and conducting business with the Hadza. However, for geographic reasons, it is more difficult, and therefore much less common, to take tourists to some of the areas inhabited by the Hadza. This allows us to use the plausibly exogenous variation in exposure due to geography to measure the impact of this cultural shock on behavior.

There are three important reasons the bias we choose to examine is the endowment effect, the tendency of people to value owned items more than non-owned items. ${ }^{1}$ First, the endowment effect is a central bias in behavioral economics because it is a direct consequence of theories of loss aversion and reference-dependent preferences, ${ }^{2}$ such as Prospect Theory. These theories are used in many applications of behavioral economics, ${ }^{3}$ and therefore the psychological underpinnings of the endowment effect have implications for a broader set of biases. Second, while theories of the evolutionary origins of the endowment effect and loss aversion have been proposed (e.g., Huck, Kirchsteiger, and Oechssler 2005; Frenkel, Heller, and Teper 2012), there are no empirical tests of these theories. Third, Hadza social life differs from that in industrialized countries in its high degree of collectivism and lack of market interactions, two factors that may plausibly affect the prevalence of the endowment effect. ${ }^{4}$ Indeed, Hadza society is characterized by a high degree of egalitarianism. Almost all of the food brought into camps is shared equally, among all camp members, regardless of kinship. While the Hadza do own some items, such as knives, bows and arrows, and animal skins, ownership is limited to what can be carried.

The results of our experiment were as follows. Hadza participants were endowed with an item, and offered to trade it for a distinct item, with both items being randomly

\footnotetext{
${ }^{1}$ In a classic experiment demonstrating this effect (Knetsch 1989), subjects in one group were endowed with a coffee mug, and given the option of trading it for a chocolate bar. A second group of subjects received a chocolate bar, which could be traded for a coffee mug. It was found that subjects in both groups decided to keep their endowed item about 90 percent of the time. In contrast, in a choice condition where subjects were asked to choose between the items, either item was chosen about 50 percent of the time.

${ }^{2}$ These include Kőszegi and Rabin (2006) and Kahneman and Tversky (1979).

${ }^{3}$ See the survey article by Camerer (2000) for field evidence in financial, labor, betting, and consumer goods markets, and the survey by Barberis and Thaler (2003) for applications in finance.

${ }^{4}$ One reason it is plausible that such factors may affect the prevalence of the endowment effect is that Maddux et al. (2010) have found cross-cultural differences in the intensity of the endowment effect. They find that students of East Asian descent experience less of an endowment effect as compared to students of Western descent, and that priming subjects with feelings of collectivism attenuates the bias.
} 
chosen by the experimenter. Hadza living in isolation were found to have no endowment effect, choosing to trade about 50 percent of the time. Our point estimate is 53 percent, and the difference from the rational benchmark of 50 percent is statistically insignificant, with a $p$-value of 0.77 . On the other hand, subjects living in the area with increased contact with modern society and markets do exhibit an endowment effect and traded their endowed good with probability of only 25 percent. The difference from 50 percent is statistically significant with a $p$-value of $<0.0001$. We test whether the results are driven by selection, experimental artifacts, or familiarity with the items used, and find it to be unlikely that these alternative hypotheses can explain the data.

Before discussing the data and experiment in detail, we briefly discuss how these findings relate to the literature. With respect to universality, our results suggest that the endowment effect is not a human universal as we found that isolated hunter-gatherers do not exhibit the bias. The only other study finding a group with no endowment effect is List's (2003) study of experienced sports cards traders suggesting the bias can be unlearned. Our study counterpoints List's, as we find that it is the population with the least market experience that behaves according to the rational benchmark. While our study displays a representative cross section of a population without an endowment effect, List's study displays a group of professionals, and highly experienced traders, who learned to avoid the bias.

Our results are particularly surprising given that the endowment effect has been documented in young children (Harbaugh, Krause, and Vesterlund 2001) and in some laboratory experiments with nonhuman primates. Notably, Chen, Lakshminarayanan, and Santos (2006) and Lakshminarayanan, Chen, and Santos (2008) have performed trading experiments showing that capuchin monkeys display a number of typically human economic behaviors, including loss aversion and the endowment effect. Other experiments involving nonhuman primates have generated mixed results. ${ }^{5}$ Perhaps the simplest explanation reconciling our results with the nonhuman primate studies that find an effect is that those results suggest very old evolutionary roots for the cognitive mechanisms necessary for the endowment effect, but that prevalence of this behavior depends on specific environmental factors, such as engaging in transactions, even if only in the laboratory. Taken together, the findings impose considerable empirical discipline on evolutionary theories of the endowment effect. Since there is more than one possible theory reconciling our findings with the previous literature, we relegate a fuller discussion of these points to the conclusion.

With respect to the influence of culture on economic preferences, the data imply that cultural and environmental factors play a key role in the presence of the endowment effect. The closest papers to ours are the economic experiments by Henrich (2000); Henrich et al. (2001, 2006) in isolated small-scale societies; and Roth et al.'s (1991) study of bargaining behavior in four countries. Our methods are complementary to theirs. Instead of comparing groups that differ across several dimensions

\footnotetext{
${ }^{5}$ Brosnan et al. (2007) reports an endowment effect in chimpanzees for food items but an opposite effect emerges for toys. Kanngiesser et al. (2011) find no endowment effect for tool items in great apes but find an effect for food items, with the exception of orangutans who do not display an endowment effect even for food items. More recently, Brosnan et al. (2012) found that chimpanzees display an endowment effect for tool items that can be immediately used for procuring food sources but not for tools with no immediate value.
} 
(e.g., Japanese versus Pittsburghers as in Roth et al. 1991; Machiguenga Indians versus Angelenos as in Henrich 2000), we analyze a plausibly exogenous cultural shock comparing two genetically and largely culturally homogeneous groups from the same population. While our shock involves exposure to different factors so that we cannot pinpoint the precise mechanism, our findings, which address concerns about omitted variable bias, reinforce the conclusion in the literature that environment can drive economic behavior.

We highlight a caveat to the interpretation of our results. One common misinterpretation of the findings is that market interactions themselves drive the presence of the endowment effect. While this theory is plausible, we stress that we are unable to isolate the exact mechanism driving the results. The Hadza were exposed not only to market interactions, such as the purchasing and selling of goods, but also to new goods and to observing Westerners interact with one another. Therefore, while the data imply that cultural factors play a role in the prevalence of the endowment effect, they do not allow one to conclude that this difference is necessarily due to higher market integration.

The paper proceeds as follows. Section I describes the experiment and data, Section II presents the results and discusses alternative explanations, and Section III concludes.

\section{Experiment and Data}

\section{A. Subjects}

Our subjects are hunter-gatherers who inhabit a savanna woodland region in a remote area of Northern Tanzania, around Lake Eyasi. The Hadza are nomadic, sleep outside under the stars, and practice neither herding nor agriculture. They live in small camps that average about 30 individuals. They are central-place foragers, meaning that foraged and hunted foods are brought back to a camp. For this reason, entire camps shift location every 4-6 weeks as resources in the immediate area are depleted. There is a sexual division of labor in which women gather fruits and dig for underground tubers and men collect honey and hunt for animals of any size, from rats to zebras. Hadza economic interactions also differ markedly from modern societies. They have no hierarchical arrangements and are egalitarian (Woodburn 1998). Decisions are made as a group. There is a high degree of sharing, so food brought to camp is divided close to evenly among all camp members (Hawkes, O'Connell, and Blurton-Jones 2001). Food is not stored and consumption is immediate. Aside from bows and arrows and the clothes on one's back, few capital goods or personal possessions exist. If one person has more than his or her share of a resource, such as two knives, the surplus will be demanded by others who have less. Thus, to a first approximation, Hadza economic life is characterized by nearly 100 percent taxation and redistribution.

One advantage of studying hunter-gatherers is that they can be used to test evolutionary theories of human behavior. Although there is considerable debate about the environment in which humans evolved, the Hadza possibly provide the best approximation for how our ancestors lived. While it is true that modern foragers may differ from early humans, they still offer the only direct observations of human behavior in the absence of agriculture. For the vast majority (over 99 percent) of our 


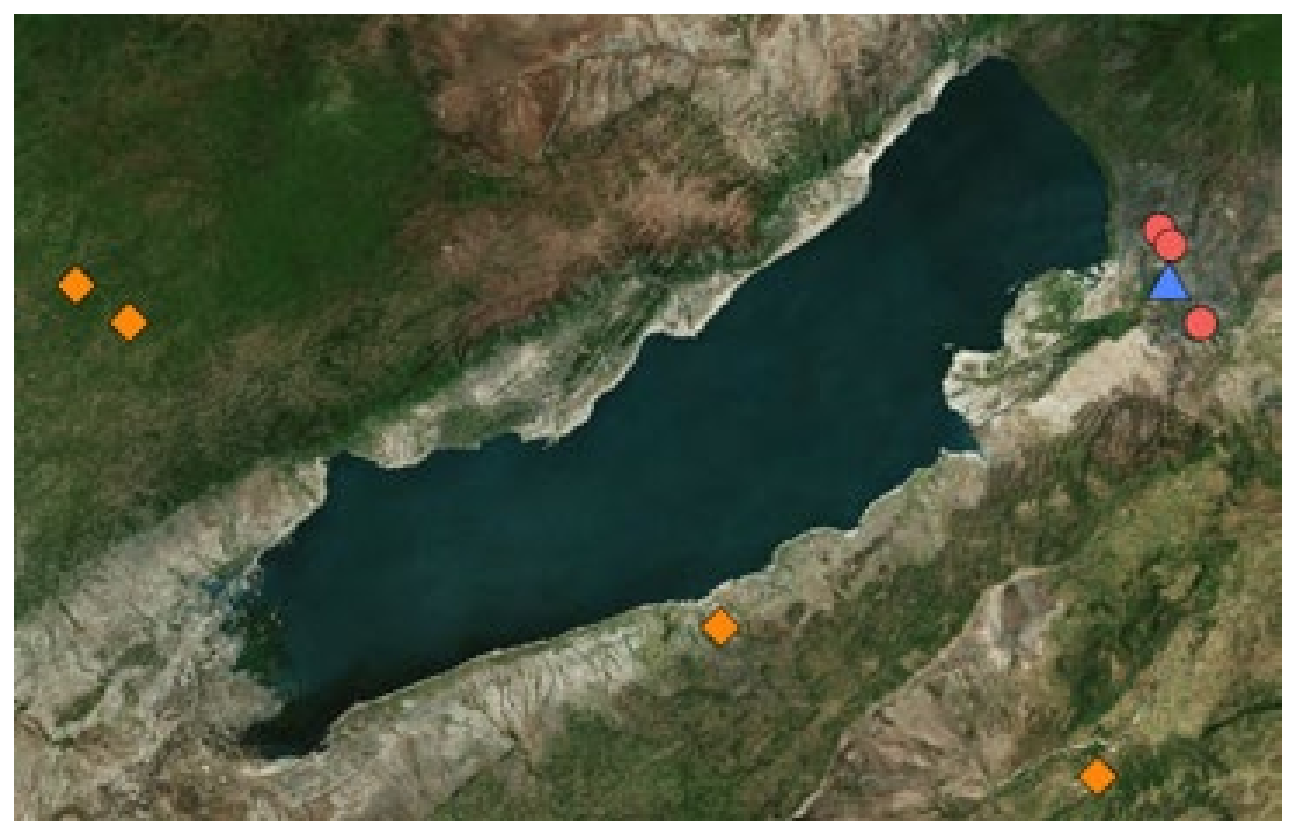

Figure 1. Region InHabited By the HadZa, ARound LaKe Eyasi

Notes: High exposure (HE) camps are marked with circles, and low exposure (LE) camps with diamonds. The triangle marks the Mangola village center.

evolutionary history, humans subsisted by means of hunting and gathering. Even around AD 1500, one-third of the planet was still inhabited by hunter-gatherers (Lee and Daly 1999). For this reason, most evolutionary theorists believe humans to be adapted to this way of life (Cosmides, Tooby, and Barkow 1992). Almost all modern human anatomy (including the brain and other physiological mechanisms involved in generating behavior) evolved when hunting and gathering was universal (Hill and Hurtado 1989). The fossil records indicate that the major increase of Homo endocranial volume, distinguishing us from our closest relatives and our Australopithecus ancestors, occurred 600-150 thousand years before present (Ruff 1997). In fact, the average endocranial volume of Homo in the early Pleistocene Epoch around 1.8 mya was $750 \mathrm{ml}$, and by 30,000 years ago when hunting and gathering dominated, it was nearly double that size (Lee and Wolpoff 2003) and has not increased since (Ruff 1997). For these reasons, it is a standard methodology in human evolutionary biology to use hunter-gatherers to test evolutionary theories of physical and behavioral traits. ${ }^{6}$

In the five years preceding our study, a subset of the Hadza has been increasingly visited by ethno-tourists. Due to its proximity to safari parks, the vast majority of the tourist visits are confined to camps northeast of Lake Eyasi, near the village of Mangola (Figure 1). The Mangola village is approximately a three hour diversion

\footnotetext{
${ }^{6}$ One problem with using a single population to test evolutionary theories is that there is considerable diversity among modern foragers. Nevertheless, the Hadza are considered a particularly good model of our past because they live in the exact location in East Africa where our early Pleistocene ancestors spent the majority of their time and because they are typical foragers along many key traits (Marlowe 2010).
} 
off a main road that connects the city of Arusha to Ngorongoro Crater. Some Hadza residing close to Mangola receive almost daily visits from tourists during the high season. In 1995, approximately one car of tourists would visit the Mangola region each week during the 3-4 month high season. In 2010, when we conducted the experiments, the number was between 10-20 cars per week (Marlowe 2010). Tour companies compensate the Hadza for these visits and for taking tourists on hunts and selling bows and arrows. In fact, Hadza in this area produce a surplus of bows and arrows, with the purpose of selling to tourists (see the online Appendix). It is not uncommon for the Hadza in the Mangola region to be found in the village center purchasing food, alcohol, or other products. In contrast, Hadza on the west and southeast of the lake rarely receive visits from tourists. To reach this group, it is necessary to drive for long distances off road and to look for the nomadic Hadza camps, a process that can take days and makes venturing far outside Mangola less appealing for commercial purposes. Henceforth, we label the two groups as high exposure (HE) and low exposure (LE).

\section{B. Experimental Procedure}

We test for the endowment effect in both the HE and LE groups using a modified version of Knetsch's (1989) study in which all subjects participated in two trials that allowed the choice to exchange an endowed item for a similar item. The first trial was between two food items (two packages of biscuits) and the second trial was between two non-food items (two lighters). The choice of items and details of the experiment were carefully chosen to address methodological concerns raised in the literature. To address concerns that the endowment effect is driven by experimental procedure (Plott and Zeiler 2007), besides the use of different items, subjects were randomly assigned, at the camp level, to one of two conditions described below.

The items were chosen to address the concern that the endowment effect may be only associated with items of evolutionary relevance, such as food, as suggested by Brosnan et al. (2007). To avoid the possibility that the endowment effect operates differently depending on the item, we conducted two separate trials with each subject. In the first trial, participants were asked to choose between two food items (biscuits), and in the second experiment, participants chose between two non-food items (lighters). We found no difference between the different item types.

The second concern is that the endowment effect may be an artifact of experimental procedures. Plott and Zeiler (2007) have found two elements to be particularly important. The first is making clear that the endowed item is randomized. This would eliminate value inferences that the subjects may make due to having received the item. The second is to prohibit subjects from holding the endowed item; this makes the transaction costs of choosing the endowed item lower. To alleviate these concerns, we randomly assigned participants to two different conditions. In condition 1, the participant physically receives an item from the experimenter and is then asked if he or she wishes to exchange it for another item. In condition 2 , the experimenter places the two items on the ground in front of the participant. She then uses a coin to randomly assign an item to the participant, who is then told that the item is his or hers. The participant is then asked whether he or she wants to trade his 
Table 1-Impact of Exposure on Probability of Trading Endowed Item

\begin{tabular}{lccccc}
\hline \hline Variable & $(1)$ & $(2)$ & $(3)$ & $(4)$ & $(5)$ \\
\hline High exposure & $-0.283^{* * *}$ & $-0.286^{*}$ & $-0.315^{* *}$ & $-0.210^{*}$ & $-0.286^{*}$ \\
$\quad$ region & $(0.0457)$ & $(0.0818)$ & $(0.0656)$ & $(0.0477)$ & $(0.0822)$ \\
Distance to & & -0.0000476 & & & 0.00144 \\
$\quad$ village $(\mathrm{km})$ & & $(0.00172)$ & & $(0.00155)$ \\
Lighter & & & & 0.0790 \\
& & & & $0.0859)$ \\
Lighter* & & & & -0.00297 \\
$\quad$ distance $(\mathrm{km})$ & & & & $0.00162)$ \\
Constant & $0.533^{* * *}$ & $0.536^{* * *}$ & $0.586^{* * *}$ & $0.438^{*}$ & $0.497 * * *$ \\
Sample & $(0.0357)$ & $(0.0818)$ & $(0.0409)$ & $(0.0477)$ & $(0.0952)$ \\
Observations & 182 & Full & Condition 1 & Condition 2 & Full \\
$R^{2}$ & 0.084 & 0.084 & 0.100 & 0.050 & 0.093 \\
\hline
\end{tabular}

Notes: Results of regressions of dependent variable equal to 1 if the subject traded endowed item, 0 otherwise. The variable high exposure region is a dummy for whether subject was in the HE camps. Lighter is a dummy for whether experiment used lighters as the items. Standard errors clustered at the camp level are reported in parentheses.

*** Significant at the 1 percent level.

** Significant at the 5 percent level.

* Significant at the 10 percent level.

or her item for the other item, and only after the subject makes a choice are they handed the chosen item. Therefore, condition 2 controls for the main experimental concerns raised by Plott and Zeiler. The wording of both conditions was carefully designed to keep subjects' expectations over their final endowment constant, as this has been found to be an important determinant of the endowment effect (Ericson and Fuster 2011). The online Appendix describes further details of the experimental procedure.

\section{Data}

Ninety-one Hadza adults, ages 16 to $70(\mathrm{M}=38.29, \mathrm{SD}=12.66)$ from eight different camps around Lake Eyasi participated in the experiment. The HE group consists of 46 individuals, ages 19 to $68(\mathrm{M}=39.20, \mathrm{SD}=11.23)$ with an approximately equal number of men (24) and women (22). The LE group had 45 individuals, ages 16 to $70(\mathrm{M}=37.36, \mathrm{SD}=14.04)$, with 21 men and 24 women. For each individual, we recorded the name of the camp where he or she resides and recorded camp locations using GPS technology. This allows us to calculate the distance of each individual to the Mangola village center. Distances range from $3.25 \mathrm{~km}$ to $81.76 \mathrm{~km}(\mathrm{M}=30.07 \mathrm{~km}$ and $\mathrm{SD}=30.99 \mathrm{~km})$. One of the subjects in the LE region reported living in a different camp in the LE region than the one in which he was found. For that reason, only seven camps are displayed in Figure 1 and panel B of Figure 2. This subject was coded as being in the LE region. For regressions involving the distance variable reported in Table 1, we used an estimated distance based on his account of nearby camps. The point estimates and standard errors are not sensitive to dropping this subject from the data. 

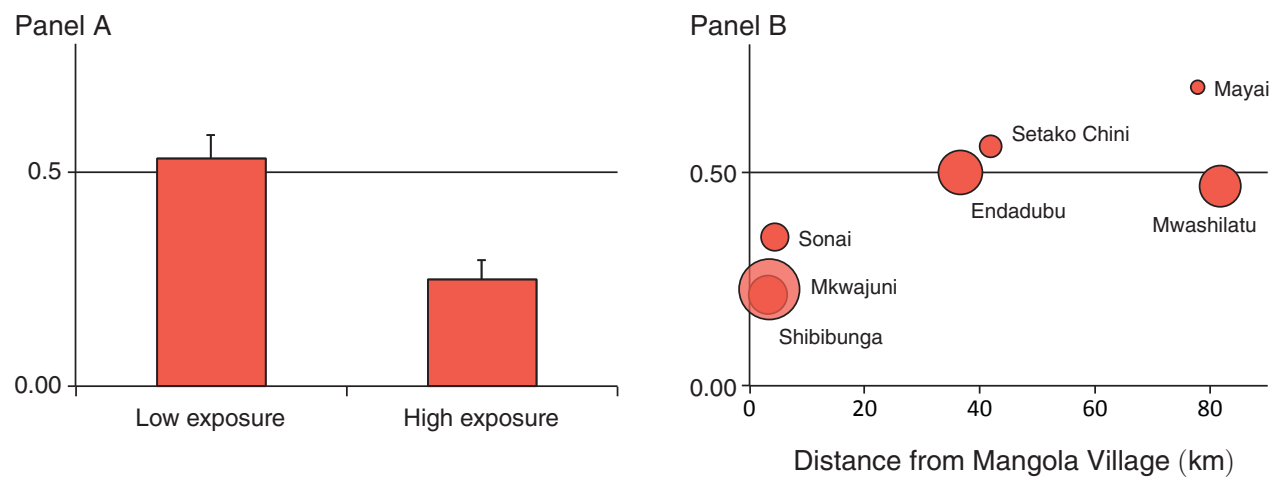

Figure 2. Fraction of Subjects Trading Endowed Item

Notes: Panel A: Fraction of subjects trading the endowed object, in low exposure and high exposure groups $(\mathrm{M}+\mathrm{SE}$ of M). Rational behavior corresponds to 0.50. Panel B: Scatter plot of the fraction of subjects trading the endowed object within each camp and distance from Mangola village in $\mathrm{km}$. Rational behavior corresponds to 0.50 . Marker size is proportional to number of subjects.

\section{Results and Alternative Explanations}

\section{A. Results}

We first examine whether the item types or treatment conditions had any effect on behavior in either the LE or HE group. Within each group, we split the sample into condition-item bins, and no differences emerged, save for a marginally significant difference between condition 1 and 2 with lighters in the LE group $(p=0.049)$. The observed direction of the difference runs antithetical to the direction expected according to the Plott and Zeiler (2007) data. The null findings are consistent with recent research that suggests that the endowment effect is driven by expected endowments (Ericson and Fuster 2011), which were kept constant across conditions in our study. We refer the reader to the online Appendix for details on the disaggregated sample, and we simply pool all treatments and conditions in the subsequent analysis.

The results of the pooled data are shown in Figure 2, panel A. In the LE group, the probability of trading the endowed item is $\mathrm{M}=0.53, \mathrm{SE}=0.05$. The 95 percent confidence interval is $[0.43,0.64]$. A one-sided binomial test of the hypothesis that the mean is 50 percent has a $p$-value 0.77 . As for the HE group, the probability of trading is $\mathrm{M}=0.25, \mathrm{SE}=0.05$. The 95 percent confidence interval is $[0.16,0.34]$, and a one-sided binomial test that the probability is 50 percent has a $p$-value of $<0.0001$. This result is robust in the disaggregated data, across the four different item-condition pairs (see Figure A.1 and Table A.2 in the online Appendix).

The results are given in regression form in Table 1, which reports results of regressions of the probability of an individual trading the endowment item on observables, with standard errors clustered at the camp level. Specification 1 is our basic model, with the only explanatory variable being whether a camp is in the HE region. The point estimate is that proximity to the village reduces the probability of trading by 28.3 percent, a highly statistically significant result. We defer discussion of the other specifications until the next section, when we test alternative explanations for the results, but highlight that the statistical significance and coefficient of the exposure 
variable are robust to a variety of controls. We note that, due to the small number of observations, the coefficient is only marginally statistically significant when distance variables are added to the model.

\section{B. Alternative Explanations}

At a first pass, the results are consistent with the view that isolated Hadza display no endowment effect and that some of the novel environmental cues in the HE region led to the emergence of the bias. We now investigate whether the data can be explained by other factors. Our main concern is self-selection of Hadza into the HE region. We also examine the possibility that the effects are driven by experimental procedures or by lack of familiarity with the goods used.

We begin by discussing the possibility of selection or the idea that Hadza who displayed the bias migrated to the HE region. Note that, because Hadza in the LE region were found to trade with probability of approximately 50 percent, for selection to fully explain the results, it is necessary that nearly all Hadza who are more attached to their possessions migrated to the HE region. There are two features of the data that make this almost perfect sorting unlikely.

First, migration across some areas of the LE and HE regions seems to be quantitatively small. Travel from the LE region on the western side of Lake Eyasi to the $\mathrm{HE}$ region on the east side of the lake is long, and many Hadza claim to have never migrated across this region. Using data from a previous social network study that examined social ties among all Hadza, through use of a comprehensive photographic database (Apicella et al. 2012), we can estimate the proportion of social ties among individuals belonging to camps from the two sides of the lake. If there were significant migration across both sides of the lake, we should expect there to be a high number of social connections between individuals on the two sides of the lake, as individuals will have left family and friends behind to migrate. However, when we examine social ties between individuals living in the three HE camps and the two LE camps on the west, only 3.8 percent of the ties were between the two sets of camps versus 96.2 percent of ties belonging within their respective camp groups. Given the low rate of social ties between these two regions and the great physical distance between them, it is unlikely that many people have migrated across this expanse. Consistent with this evidence, we find that our sample is balanced in observables like age and sex, which could plausibly be related to migratory behavior. Overall, it is quantitatively implausible that migration can explain the fraction of subjects trading changing from 53 percent to 25 percent.

Second, the strength of the endowment effect depends on whether a camp is in the Mangola region but not on the distance to the village itself. Figure 2, panel B plots the fraction of subjects trading in each camp versus the distance to the village center. The figure reveals that the fraction of subjects trading is approximately 25 percent for camps close to the village. However, this probability is close to 50 percent for camps far from the village, regardless of the distance. This is confirmed by a regression of the probability of trading on a dummy for the Mangola region and distance from the village, which finds that distance is statistically insignificant. Moreover, controlling for distance does not considerably affect the estimated coefficient of the dummy for the HE region (Table 1, specifications 1 and 2). These results are hard to 
reconcile with the selection hypothesis because being closer to the village reduces the cost of migration. If selection were driving the results, we would expect fewer subjects to migrate in the farthest camps, finding a negative effect of distance on the probability of trading. This is not the case.

Finally, we emphasize that the Hadza display complex residential and migratory patterns, which emphasize bilateral kin associations, affinal alliances, and frequent brother-sister affiliation (Hill et al. 2011). The perfect sorting necessary for selection to explain the results is unlikely to be consistent with these patterns.

Another concern is that results are driven by an artifact of experimental procedure. That is, that our experiment is not measuring the endowment effect but a differential response of the two groups to some experimental artifact. To test whether this is the case, we split the sample into the two conditions and regressed the dummy for trading on the dummy for the HE region. The HE dummy's coefficient is stable and remains statistically significant across these two subsamples (Table 1, specifications 3 and 4). Condition 2 eliminates the two main experimental procedures that were found by Plott and Zeiler $(2005,2007)$ to drive the endowment effect. Because eliminating these procedures did not considerably affect the coefficient of the HE dummy, it seems unlikely that experimental artifacts can drive the 25 percent difference between the HE and LE groups.

Finally, another alternative explanation is that the cultural shock in the HE region did matter but that its effect was due to increased familiarity of subjects in the HE region with the items. While we are not familiar with any studies documenting the effect of familiarity on the endowment effect, this is in principle a plausible explanation. In fact, standard narrow framing and loss aversion theories imply no endowment effect if unfamiliar items are in the same consumption dimension. For example, consider a subject offered to trade an endowed copy of War and Peace for Anna Karenina. If the subject views parting with War and Peace as a loss, and receiving Anna Karenina as a gain, loss aversion reduces her willingness to trade. However, were the subject offered the Russian edition of these books, she might simply view them in the consumption dimension "books in Russian." As such, trading would involve no net gain or loss, and loss aversion would not reduce the willingness to trade.

There are three features of the data and environment that are inconsistent with this explanation. First, conditional on whether a camp is in the HE or LE region, the strength of the endowment effect does not depend on distance to the village. Items such as biscuits and lighters do occasionally make their way to the LE region, be it from Hadza traveling or carried by researchers. However, the frequency with which these items make it to the LE region should be correlated with distance. This would imply an increasing relationship between the probability of trading and distance, contrary to the negative, economically negligible, and statistically insignificant relationship in the data (Figure 2, panel B and Table 1, specification 2). The point estimate is that a $100 \mathrm{~km}$ increase in distance reduces the probability of trading by 0.5 percent. Second, being a durable good, lighters are considerably more likely to be found in the LE region. However, we found no differences between goods or significant coefficients for the interaction of distance to the village and lighters being used (Table 1, specification 5). Third, the gap in familiarity across the two groups is likely too small to explain the stark difference in trading probability. Lighters, and these particular biscuits, were not available for sale in dukas (small stands) in 
Mangola when this study was conducted. At the same time, Hadza in both regions value the goods used. Because the Hadza are often hungry and enjoy sweet tastes, the biscuits are highly valued. While the Hadza have limited experience with packaged foods, they do have some experience with biscuits and often ask for sweet foods brought by researchers. Likewise, lighters are valued due to their convenience in making fires compared with the more tedious fire drill procedure. Women claim not to be able to make fire using drills and even men prefer to use matches or lighters to avoid this chore (Marlowe 2010).

\section{Conclusion}

We conclude by relating our findings to the three questions we posed in the introduction. We find that an isolated population of hunter-gatherers displays no endowment effect, while a subset of this group with increased contact with modern society and markets displays the bias. The robust implications of the data are that, first, the endowment effect bias is not a universal behavior because isolated Hadza do not display the bias. Second, regardless of the exact mechanism driving behavior in the HE group, culture can lead to differences in the presence of the endowment effect. Finally, to the extent that present-day hunter-gatherers, and specifically the Hadza, can be used to model the behavior of our ancestors, our results suggest that early humans did not display the bias.

While the standard explanation for the endowment effect is that it is a manifestation of loss aversion (Kahneman and Tversky 1979) other explanations have been offered. Most recently, it has been suggested that the endowment effect results from the reluctance to trade on unfavorable terms with respect to reference prices (Weaver and Frederick 2012). This explanation requires that, for individuals to have an endowment effect, they must first have notions of reference prices or exchange rates, which come from market experience. Our finding that the endowment effect is not present in isolated hunter-gatherers living in a non-monetary subsistence economy provides necessary, though not sufficient, evidence for this explanation.

Our finding that isolated hunter-gatherers do not exhibit the endowment effect is somewhat surprising given the robustness of the endowment effect in industrialized societies and evidence that the endowment effect is present in young American children and some nonhuman primates (Harbaugh, Krause, and Vesterlund 2001; Brosnan et al. 2007; Kanngiesser et al. 2011; Lakshminarayanan, Chen, and Santos 2008). With respect to young children, one possible explanation is that American children are exposed to concepts of ownership, the use of money, and compensation for work at very young ages and even limited exposure to money does affect behavior (Vohs, Mead, and Goode 2006). While the data suggesting that nonhuman primates possess an endowment effect is mixed, we offer two explanations that help reconcile our findings with those studies that report an effect. One possible explanation is that the same environmental circumstances driving the endowment effect in humans may also be driving the endowment effect in these primates. These studies are conducted in the laboratory where the animals are explicitly trained to interact in token economies. In fact, a wide range of economic behaviors, such as responding to prices and advertisements, have been demonstrated in the nonhuman laboratory primates. While these findings suggest that animals have the capacity to display 
these behaviors, it is unclear whether they would occur naturally, as animals must first be trained to participate in these experiments. A second plausible explanation is that the endowment effect is an evolutionary ancient trait, present in the last common ancestor of capuchins and humans, but is suppressed by cultural features of hunter-gatherer life. Whatever the explanation, our results point to the role of culture in generating differences.

Our findings dovetail with a compelling line of cross-cultural research that suggests that cultural factors, and in particular the degree of market integration, influence economic preferences. One advantage of our methodology over traditional comparative studies is that we compare two genetically and largely culturally homogeneous groups, eliminating the many additional confounds that are present when data are gathered across societies. That said, we are unable to isolate the exact mechanism(s). Along with increased market integration, the HE group was exposed to concepts of ownership, selling and purchasing of goods, and payment for labor, all of which could be driving the group's preference for owned items. Thus, while it is unlikely that the findings are explained by self-selection or experimental artifacts, the data do not allow us to infer the precise mechanism responsible for the difference between the two groups.

\section{REFERENCES}

Apicella, Coren L., Eduardo M. Azevedo, Nicholas A. Christakis, and James H. Fowler. 2014. "Evolutionary Origins of the Endowment Effect: Evidence from Hunter-Gatherers: Dataset." American Economic Review. http://dx.doi.org/10.1257/aer.104.6.1793.

Apicella, Coren L., Frank W. Marlowe, James H. Fowler, and Nicholas A. Christakis. 2012. "Social Networks and Cooperation in Hunter-Gatherers." Nature 481 (7382): 497-501.

Barberis, Nicholas, and Richard Thaler. 2003. "A Survey of Behavioral Finance." In Handbook of the Economics of Finance, Vol. 1B, edited by George M. Constantinides, Milton Harris, and Rene Stulz, 1053-1128. Amsterdam: Elsevier.

Brosnan, Sarah F., Owen D. Jones, Molly Gardner, Susan P. Lambeth, and Steven J. Schapiro. 2012. "Evolution and the Expression of Biases: Situational Value Changes the Endowment Effect in Chimpanzees." Evolution and Human Behavior 33 (4): 378-86.

-Brosnan, Sarah F., Owen D. Jones, Susan P. Lambeth, Mary Catherine Mareno, Amanda S. Richardson, and Steven J. Schapiro. 2007. "Endowment Effects in Chimpanzees." Current Biology 17 (19): 1704-07.

Camerer, Colin F. 2000. "Prospect Theory in the Wild: Evidence from the Field." In Choices, Values, and Frames, edited by Daniel Kahneman and Amos Tversky, 288-300. Cambridge, UK: Cambridge University Press.

Chen, M. Keith, Venkat Lakshminarayanan, and Laurie R. Santos. 2006. "How Basic Are Behavioral Biases? Evidence from Capuchin Monkey Trading Behavior." Journal of Political Economy 114 (3): 517-37.

Cosmides, Leda, John Tooby, and Jerome H. Barkow. 1992. "Introduction: Evolutionary Psychology and Conceptual Integration." In The Adapted Mind: Evolutionary Psychology and the Generation of Culture, edited by Jerome H. Barkow, Leda Cosmides, and John Tooby, 3-15. New York: Oxford University Press.

Frenkel, Sivan, Yuval Heller, and Roee Teper. 2012. "Endowment as a Blessing." Unpublished.

Harbaugh, William T., Kate Krause, and Lise Vesterlund. 2001. "Are Adults Better Behaved Than Children? Age, Experience, and the Endowment Effect." Economics Letters 70 (2): 175-81.

-Hawkes, K., J. F. O’Connell, and N. G. Blurton Jones. 2001. "Hadza Meat Sharing." Evolution and Human Behavior 22 (2): 113-42.

-Henrich, Joseph. 2000. "Does Culture Matter in Economic Behavior? Ultimatum Game Bargaining among the Machiguenga of the Peruvian Amazon." American Economic Review 90 (4): 973-79.

-Henrich, Joseph, Robert Boyd, Samuel Bowles, Colin Camerer, Ernst Fehr, Herbert Gintis, and Richard McElreath. 2001. "In Search of Homo Economicus: Behavioral Experiments in 15 Small-Scale Societies.” American Economic Review 91 (2): 73-78. 
Henrich, Joseph, Richard McElreath, Abigail Barr, Jean Ensminger, Clark Barrett, Alexander Bolyanatz, Juan Camilo Cardenas, et al. 2006. "Costly Punishment across Human Societies.” Science 312 (5781): 1767-70.

Hill, Kim, and A. Magdalena Hurtado. 1989. "Hunter-Gatherers of the New World." American Scientist 77 (5): 436-43.

Hill, Kim R., Robert S. Walker, Miran Božičevic, James Eder, Thomas Headland, Barry Hewlett, A. Magdalena Hurtado, Frank W. Marlowe, Polly Wiessner, and Brian Wood. 2011. "Co-Residence Patterns in Hunter-Gatherer Societies Show Unique Human Social Structure.” Science 331 (6022): 1286-89.

Huck, Steffen, Georg Kirchsteiger, and Jorg Oechssler. 2005. "Learning to Like What You HaveExplaining the Endowment Effect.” Economic Journal, 115 (505): 689-702.

Kahneman, Daniel, and Amos Tversky. 1979. "Prospect Theory: An Analysis of Decision under Risk." Econometrica 263-91.

Kanngiesser, Patricia, Laurie R. Santos, Bruce M. Hood, and Josep Call. 2011. "The Limits of Endowment Effects in Great Apes (Pan Paniscus, Pan Troglodytes, Gorilla Gorilla, Pongo Pygmaeus)." Journal of Comparative Psychology 125 (4): 436-45.

Knetsch, Jack L. 1989. "The Endowment Effect and Evidence of Nonreversible Indifference Curves." American Economic Review 79 (5): 1277-84.

Köszegi, Botond, and Matthew Rabin. 2006. "A Model of Reference-Dependent Preferences." Quarterly Journal of Economics 121 (4): 1133-65.

Lakshminarayanan, Venkat, M. Keith Chen, and Laurie R. Santos. 2008. "Endowment Effect in Capuchin Monkeys." Philosophical Transactions of the Royal Society B: Biological Sciences 363 (1511): 3837-44.

Lee, Richard B., and Richard H. Daly. 1999. The Cambridge Encyclopedia of Hunters and Gatherers. New York: Cambridge University Press.

Lee, Sang-Hee, and Milford H. Wolpoff. 2003. "The Pattern of Pleistocene Human Brain Size Evolution." Paleobiology 29 (2): 185-96.

-List, John A. 2003. "Does Market Experience Eliminate Market Anomalies?" Quarterly Journal of Economics 118 (1): 41-71.

Maddux, William W., Haiyang Yang, Carl Falk, Hajo Adam, Wendi Adair, Yumi Endo, Ziv Carmon, and Steven J. Heine. 2010. "For Whom is Parting with Possessions More Painful? Cultural Differences in the Endowment Effect." Psychological Science 21 (12): 1910-17.

Marlowe, Frank W. 2010. The Hadza: Hunter-Gatherers of Tanzania. Berkeley: University of California Press.

Marzilli Ericson, Keith M., and Andreas Fuster. 2011. "Expectations as Endowments: Evidence on Reference-Dependent Preferences from Exchange and Valuation Experiments." Quarterly Journal of Economics 126 (4): 1879-1907.

Plott, Charles R., and Kathryn Zeiler. 2005. "The Willingness to Pay-Willingness to Accept Gap, the 'Endowment Effect,' Subject Misconceptions, and Experimental Procedures for Eliciting Valuations." American Economic Review 95 (3): 530-45.

Plott, Charles R., and Kathryn Zeiler. 2007. "Exchange Asymmetries Incorrectly Interpreted as Evidence of Endowment Effect Theory and Prospect Theory?" American Economic Review 97 (4): 1449-66.

- Rayo, Luis, and Gary S. Becker. 2007. "Evolutionary Efficiency and Happiness.” Journal of Political Economy 115 (2): 302-37.

Robson, Arthur J., and Larry Samuelson. 2009. "The Evolution of Time Preference with Aggregate Uncertainty." American Economic Review 99 (5): 1925-53.

Roth, Alvin E., Vesna Prasnikar, Masahiro Okuno-Fujiwara, and Shmuel Zamir. 1991. "Bargaining and Market Behavior in Jerusalem, Ljubljana, Pittsburgh, and Tokyo: An Experimental Study." American Economic Review 81 (5): 1068-95.

Ruff, Christopher B., Erik Trinkaus, and Trenton W. Holliday. 1997. "Body Mass and Encephalization in Pleistocene Homo." Nature 387 (6629): 173-76.

- Vohs, Kathleen D., Nicole L. Mead, and Miranda R. Goode. 2006. "The Psychological Consequences of Money." Science 314 (5802): 1154-56.

-Weaver, Ray, and Shane Frederick. 2012. "A Reference Price Theory of the Endowment Effect." Journal of Marketing Research 49 (5): 696-707.

Woodburn, James. 1998. "'Sharing is Not a Form of Exchange': An Analysis of Property-Sharing in Immediate-Return Hunter-Gatherer Societies." In Property Relations: Renewing the Anthropological Tradition, edited by C. M. Hann, 48-63. Cambridge, UK: Cambridge University Press. 